\title{
Porcine deltacoronavirus: histological lesions and genetic characterization
}

\author{
Leyi Wang $^{1} \cdot$ Jeff Hayes $^{1} \cdot$ Craig Sarver $^{1} \cdot$ Beverly Byrum $^{1} \cdot$ Yan Zhang $^{1}$
}

Received: 23 June 2015/Accepted: 24 September 2015/Published online: 16 October 2015

(C) Springer-Verlag Wien 2015

\begin{abstract}
First identified in 2012 in a surveillance study in Hong Kong, porcine deltacoronavirus (PDCoV) is a proposed member of the genus Deltacoronavirus of the family Coronaviridae. In February of 2014, PDCoV was detected in pigs with clinical diarrheal symptoms for the first time in the USA. Since then, it has been detected in more than 20 states in the USA and in other countries, including Canada, South Korea, and mainland China. So far, histological lesions in the intestines of pigs naturally infected with PDCoV under field conditions have not been reported. In this report, we describe the characteristic histological lesions in the small intestine that were associated with PDCoV infection, as evidenced by detection of viral nucleic acid by RT-PCR. In addition, we performed genomic analysis to determine the genetic relationship of all PDCoV strains from the four countries. We found that PDCoV mainly caused histological lesions in the small intestines of naturally infected piglets. Sequence analysis demonstrated that the PDCoV strains of different countries are closely related and shared high nucleotide sequence similarity; however, deletion patterns in the spike and 3' untranslated regions are different among the strains from mainland China, Hong Kong, the USA, and South Korea. Our study highlights the fact that continual surveillance is needed to trace the evolution of this virus.
\end{abstract}

Leyi Wang

wang.1074@buckeyemail.osu.edu

Yan Zhang

yzhang@agri.ohio.gov

1 Animal Disease Diagnostic Laboratory, Ohio Department of Agriculture, 8995 East Main Street, Building \#6, Reynoldsburg, $\mathrm{OH} 43068$, USA
Deltacoronavirus is a newly established genus in the family Coronaviridae in which there are three officially recognized species to date. One proposed member of this genus is porcine deltacoronavirus (PDCoV) $[1,15]$. PDCoV has the following genome arrangement: 5' untranslated region (UTR), open reading frame $1 \mathrm{a} / \mathrm{lb}(\mathrm{ORF} 1 \mathrm{a} / \mathrm{lb})$, spike (S), envelope (E), membrane (M), nonstructural protein 6 (NS6), nucleocapsid (N), nonstructural protein 7 (NS7), and 3' UTR [15]. PDCoV was first discovered in pig rectal swabs in a surveillance study in Hong Kong in 2012 [15]. This surveillance study showed that 17 out of 169 samples tested positive for PDCoV. However, the clinical significance of this virus was not clear. In February 2014, fecal samples from sows with watery diarrhea and intestinal samples from piglets that died from scour on a pig farm in Ohio were submitted for porcine epidemic diarrhea virus (PEDV) testing. All samples were negative for PEDV by reverse transcription (RT) PCR assay [12]. Further testing ruled out other known viral pathogens, including transmissible gastroenteritis virus (TGEV), rotaviruses and bacterial pathogens [12]. However, the clinical signs were consistent with porcine epidemic diarrhea (PED) or transmissible gastroenteritis (TGE), with mortality ranging from 30 to $50 \%$ in piglets. Electron microscopic observations also suggested a coronavirus-like virus as a causal agent [12]. Subsequently, PDCoV was detected from all samples [12]. Since then, PDCoV has been detected in over 20 states of the United States (US), Canada, and South Korea [6, 10, $11,13,14]$. Recently, PDCoV has been reported in several provinces of mainland China [3]. It has been reported that pigs were infected by either PDCoV alone or together with other porcine diarrheal viruses, including PEDV or porcine rotaviruses.

To determine whether PDCoV fulfills Koch's postulates, three research groups have recently conducted animal 

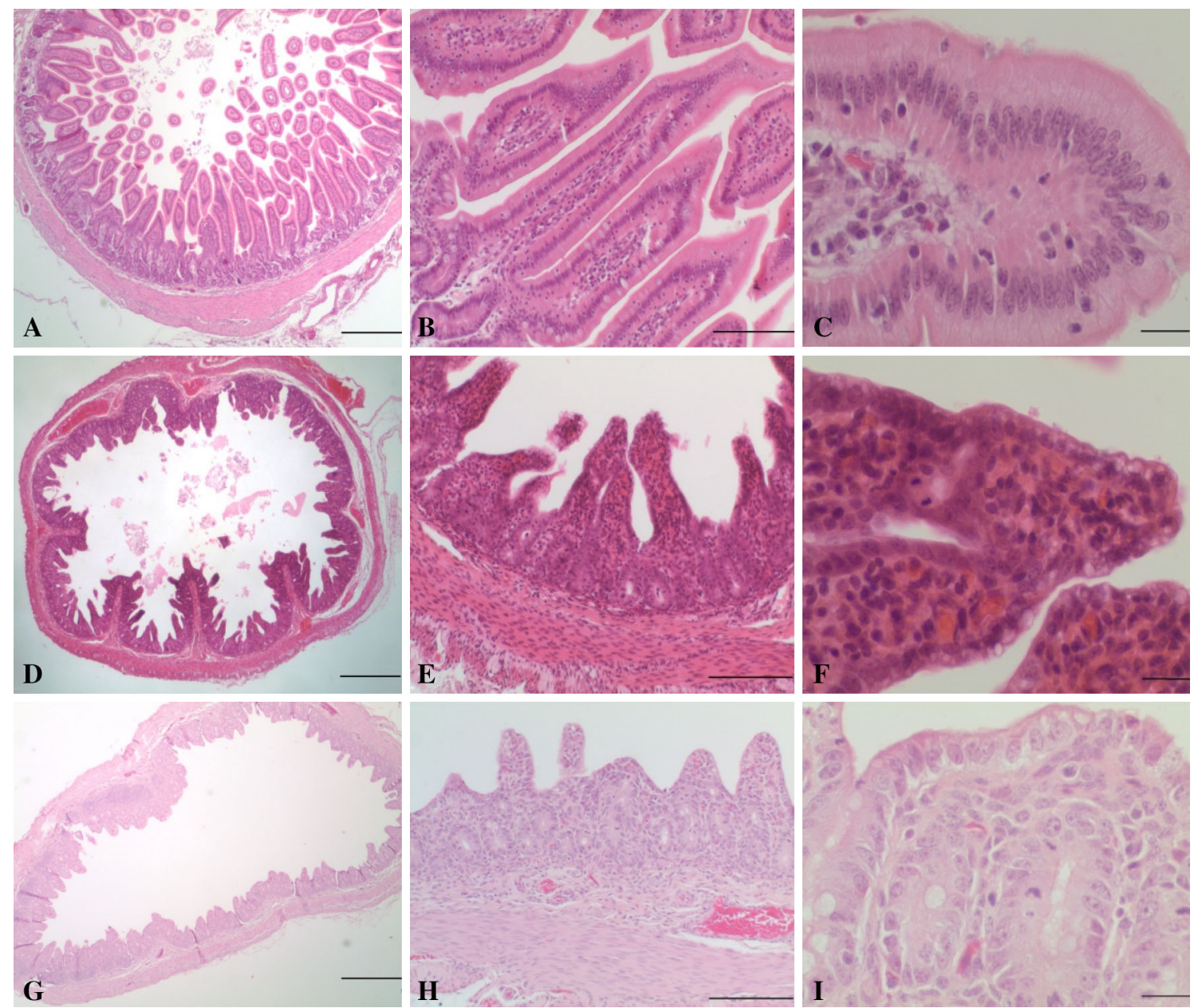

Fig. 1 Photomicrographs depicting jejunum sections from nursing piglets not infected with a coronavirus (A-C), infected with porcine deltacoronavirus (PDCoV) (D-F), and infected with porcine epidemic diarrhea virus (PEDV) (G-I). A, jejunum; note villi of normal length (villus:crypt ratios >3-4:1). Bar $=500 \mu \mathrm{m}$. B, jejunum; villi are of normal length and are lined by tall columnar superficial enterocytes. Bar $=100 \mu \mathrm{m}$. C, jejunum; high magnification of superficial enterocytes with abundant pale eosinophilic cytoplasm. Bar $=20 \mu \mathrm{m}$. D, jejunum; moderate blunting of villi increasing the diameter of clear luminal space. Bar $=500 \mu \mathrm{m}$. E, jejunum; note decreased villus:crypt ratios (between 2:1-3:1) with fusion of two villi in the center of the

challenge studies using gnotobiotic and conventional pigs and found that PDCoV could reproduce the diarrheal disease and cause histopathological lesions including acute diffuse, severe atrophic enteritis [2, 5, 7], further supporting the clinical observations suggesting its role in swine diarrhea. However, histologic lesions from natural infection have not been reported. In the current study, histological lesions in piglets infected with PDCoV are presented. In addition, sequence analysis of the PDCoV strains from different countries is described.

Three fecal samples and four piglets were collected from an Indiana (IN2847) pig farm experiencing an outbreak of image. Small amounts of cellular debris are evident in the lumen of scattered crypts. Bar $=100 \mu \mathrm{m}$. F, jejunum; superficial enterocytes have cuboidal morphology, and the cytoplasm is often vacuolated. Note villous fusion as well as a mitotic figure in the crypt epithelial cell. Bar $=20 \mu \mathrm{m}$. G, jejunum; severe diffuse villous atrophy with frequent fusion of villi. Bar $=500 \mu \mathrm{m}$. $\mathrm{H}$, jejunum; marked villous atrophy with villus:crypt ratios of 1:1-2:1. Marked cuboidal attenuation of superficial enterocytes is present. Bar $=100 \mu \mathrm{m}$. I, jejunum; cytoplasmic vacuolation of attenuated superficial enterocytes is shown, as are mitotic figures in crypt epithelial cells. Note the collapse of the lamina propria stroma. Bar $=20 \mu \mathrm{m}$

diarrhea and were submitted to the Animal Disease Diagnostic Laboratory (ADDL) of the Ohio Department of Agriculture. Fresh and formalin-fixed intestine samples were collected for testing.

Fecal and fresh intestinal samples were processed for extracting viral RNA using a MagMAX Pathogen RNA/DNA Kit (Applied Biosystems ${ }^{\mathrm{TM}}$, Life Technologies). Real-time reverse transcription (RT) PCR assays for PEDV and PDCoV were used to test the nucleic acid samples. The results showed that all samples of feces and intestines from case IN2847 were positive for $\mathrm{PDCoV}$, with a $\mathrm{Ct}$ value 19 , but all of the samples from this case were negative for PEDV. 
Fig. 2 Phylogenetic tree constructed on the basis of the whole-genome nucleotide sequences of 23 porcine deltacoronavirus (PDCoV) strains from different countries. The dendrogram was constructed using the neighborjoining method in the MEGA software package, version 6.05 (http://www.megasoftware.net). Bootstrap resampling $(1,000$ replications) was performed, and bootstrap values are indicated for each node. Reference sequences obtained from GenBank are indicated by strain name. The scale bar represents 0.01 nucleotide substitutions per site

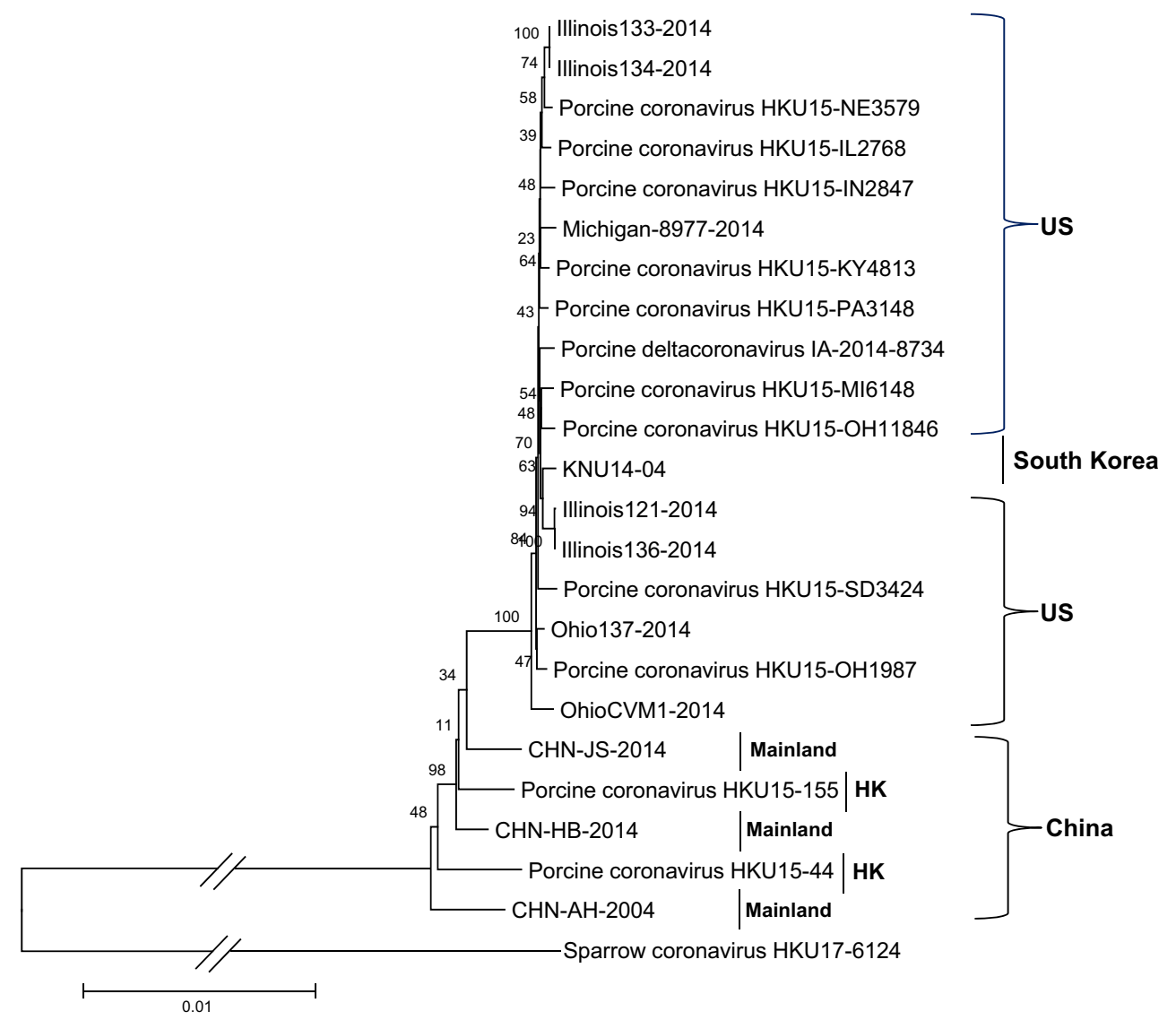

The histological lesions of the formalin-fixed intestines of the four piglets were consistent with those observed in animal challenge studies [2, 5, 7]. In comparison with jejunum sections of normal nursing piglets from a different farm (Fig. 1A-C), microscopic examination of the small intestines of piglets infected with PDCoV revealed multifocal to diffuse villous atrophy and villous attenuation with villus-to-crypt ratios of 2-3:1, and with fusion of some villi (Fig. 1D and E). The enterocytes of affected villi in the infected piglets had cuboidal to flattened squamous morphology (attenuation); enterocytes often had a vacuolated cytoplasm (Fig. 1F). The lamina propria contained small numbers of necrotic cells and nuclear debris, and small numbers of lymphocytes and eosinophils (stromal condensation) (Fig. 1F). In scattered crypts, there were increased numbers and layers of crypt epithelial cells with multiple mitotic figures (hyperplasia). Scattered submucosal areas were distended with clear spaces (edema), and the intestinal vasculature was moderately congested. There were no significant microscopic lesions in the colon and rectum. Histopathologic alterations in small-intestinal sections were similar to but less severe than those observed in piglets affected with PEDV observed at the ADDL and reported by others $[4,8]$. Attenuation and cytoplasmic vacuolation of superficial enterocytes was similar to that observed in PEDV-infected pigs, but villous atrophy and villous fusion were not as severe as seen in PEDV infection (Fig. 1G-1I).

To analyze the phylogenetic relationships among PDCoV isolates from different countries, we collected all full-length nucleotide sequences of PDCoV in GenBank, including one newly deposited sequence (OH11846) from our laboratory. All sequences were aligned using the MEGA6 program, and phylogenetic trees were constructed with MEGA6 using the neighbor-joining method. The reliability of the branching orders was evaluated by the bootstrap test $(\mathrm{n}=1,000)$. Phylogenetic analysis indicated that OH11846 is closely related to IN2847 and other porcine deltacoronavirus strains from the US, South Korea, and China belonging to the genus Deltacoronavirus (Fig. 2). A high level of nucleotide sequence similarity (> $98 \%$ ) was observed among the PDCoV strains from different countries, supporting the previous assumption that a single genotype of PDCoV is currently circulating in the US [13].

Further sequence analysis showed that, in comparison to strain HKU15-44, strain HKU15-155 has two 3-nt deletions in the S gene and 3' UTR (Fig. 3). Similar to HKU1544, the strains from the US and South Korea do not have these deletions. However, the three strains from China have 
Porcine coronavirus_HKU15-44 Porcine_coronavirus_HKU15-155

Spike

$\mathrm{CHN}-\mathrm{AH}-2004$

CHN-HB-2014

CHN-JS-2014

Illinois121-2014

Illinois133-2014

Illinois134-2014

Illinois136-2014

KNU14-04

Michigan-8977-2014

Ohio137-2014

OhioCVM1-2014

Porcine coronavirus_HKU15-PA3148

Porcine_coronavirus_HKU15-IL2768

Porcine_coronavirus_HKU15-IN2847

Porcine_coronavirus_HKU15-KY4813

Porcine coronavirus_HKU15-MI6148

Porcine_coronavirus_HKU15-NE3579

Porcine_coronavirus_HKU15-OH1987

Porcine_coronavirus_HKU15-OH11846

Porcine coronavirus_HKU15-SD3424

Porcine_deltacoronavirus_IA-2014-8734

Porcine_coronavirus_HKU15-44

3' UTR

Porcine_coronavirus_HKU15-155

$-\mathrm{AH}-200$

CHN-HB-2014

CHN-JS-2014

Illinois121-2014

Illinois133-2014

Illinois134-2014

Illinois136-2014

KNU14-04

Michigan-8977-2014

Ohio137-2014

OhioCvM1-2014

Porcine_coronavirus_HKU15-IL2768

Porcine_coronavirus_HKU15-IN2847

Porcine_coronavirus_HKU15-KY4813

Porcine coronavirus_HKU15-MI6148

Porcine_coronavirus_HKU15-NE3579

Porcine_coronavirus_HKU15-OH11846

Porcine_coronavirus_HKU15-OH1987

Porcine coronavirus HKU15-PA314

Porcine_coronavirus_HKU15-SD3424

Porcine_deltacoronavirus_IA-2 014-8734

Fig. 3 Sequence alignments of partial spike genes and 3' untranslated regions (UTRs) of different porcine deltacoronavirus strains, performed using the MUSCLE program in the MEGA software package. Red rectangles indicate the 3 -nt deletion sites. The first

different deletion patterns. Strains CHN-HB-2014 and CHN-JS-2014 have a 3-nt deletion in the S gene only, while $\mathrm{CHN}-\mathrm{AH}-2004$ strain has a 3-nt deletion in the 3' UTR only (Fig. 3). Compared with that of HKU15-155, the 3-nt deletion site in strain CHN-AH-2004 is shifted one nucleotide to the right. The 3-nt deletion in the spike gene of HKU15-155, CHN-HB-2014, and CHN-JS-2014 results in the deletion of one amino acid (asparagine) without causing a frameshift (Fig. 3). In addition to the two 3-nt deletions observed in the S gene and the 3' UTR, there are a total of 689 point mutations, including 533 silent mutations (5' UTR, 8; ORF1a, 211; ORF1b, 156; S, 100; E, 5; M, 16; NS6, 3; N, 25; NS7, 4; 3' UTR, 5) and 156 nonsilent mutations (ORF1a, 60; ORF1b, 26; S, 41; E, 0; M, 1; NS6, 3; N, 9; NS7, 16) in the full-length genome sequence of PDCoV. It remains unknown whether the strains with one or two 3-nt deletions were derived from strains without deletions, or vice versa. The origin of this virus in the US is

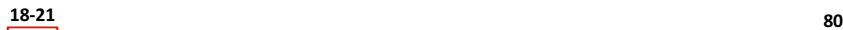

18-21 80

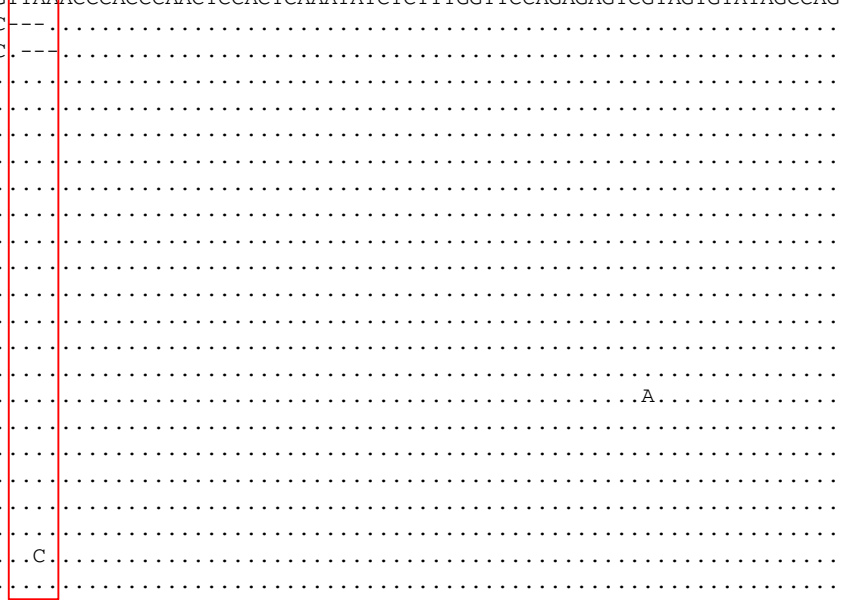

nucleotide of each codon is shown in bold in the spike alignment. A dot indicates that the nucleotide exactly matches the strain HKU1544. A dash indicates a nucleotide deletion compared to the strain HKU15-44 (color figure online)

also unknown, but it was most likely imported from China by the same route of transmission that was used by PEDV. This virus was then subsequently transported to Canada and South Korea. PDCoV was first reported in Hong Kong of China and the US in 2012 and 2014, respectively. Recent retrospective studies showed that PDCoV could have been circulating in the field a few years before identification and detection [9].

In conclusion, our data from piglets naturally infected with PDCoV are consistent with the findings of recent pathogenesis challenge studies in pigs $[2,5,7]$. Histopathologic findings in those studies and the present case show that PDCoV causes characteristic microscopic lesions that are similar to but milder than those caused by PEDV. Although different deletion patterns were observed in the S gene and 3' UTR of the strains from China and other countries, including the US and South Korea, this study suggests that the PDCoV strains circulating in the 
different countries belong to a single genotype. Further study is needed to determine the origin of PDCoV and the evolution of this virus in pig populations.

Acknowledgments The authors thank Jason Herr and Kerri Lawrence for technical assistance.

\section{Compliance with ethical standards}

Conflict of interest The authors declare that they have no conflict of interest.

\section{References}

1. Adams MJ, Carstens EB (2012) Ratification vote on taxonomic proposals to the International Committee on Taxonomy of Viruses (2012). Arch Virol 157:1411-1422

2. Chen Q, Gauger P, Stafne M, Thomas J, Arruda P, Burrough E, Madson D, Brodie J, Magstadt D, Derscheid R, Welch M, Zhang J (2015) Pathogenicity and pathogenesis of a United States porcine deltacoronavirus cell culture isolate in 5-day-old neonatal piglets. Virology 482:51-59

3. Feng L (2014) The updated epidemic and controls of swine enteric coronavirus in China. Presentation at the international conference on swine enteric coronavirus diseases, Chicago, USA, Sep 23-25, 2014

4. Jung K, Wang Q, Scheuer KA, Lu Z, Zhang Y, Saif LJ (2014) Pathology of US porcine epidemic diarrhea virus strain PC21A in gnotobiotic pigs. Emerg Infect Dis 20:662-665

5. Jung K, Hu H, Eyerly B, Lu Z, Chepngeno J, Saif LJ (2015) Pathogenicity of 2 porcine deltacoronavirus strains in gnotobiotic pigs. Emerg Infect Dis 21:650-654
6. Lee S, Lee C (2014) Complete genome characterization of Korean porcine deltacoronavirus strain KOR/KNU14-04/2014. Genome Announc 2. doi:10.1128/genomeA.01191-14

7. Ma Y, Zhang Y, Liang X, Lou F, Oglesbee M, Krakowka S, Li J (2015) Origin, evolution, and virulence of porcine deltacoronaviruses in the United States. mBio 6:e00064

8. Stevenson GW, Hoang H, Schwartz KJ, Burrough ER, Sun D, Madson D, Cooper VL, Pillatzki A, Gauger P, Schmitt BJ, Koster LG, Killian ML, Yoon KJ (2013) Emergence of Porcine epidemic diarrhea virus in the United States: clinical signs, lesions, and viral genomic sequences. J Vet Diagn Invest 25:649-654

9. Thachil A, Gerber PF, Xiao CT, Huang YW, Opriessnig T (2015) Development and application of an ELISA for the detection of porcine deltacoronavirus IgG antibodies. PLoS One 10:e0124363

10. ThePigSite (2014) Swine deltacoronavirus detected on Canadian pig farms. http://www.thepigsite.com/swinenews/36095/swinedeltacoronavirus-detected-on-canadian-pig-farms/. Accessed 26 May 2015

11. USDA (US Department of Agriculture) (2015) Swine enteric coronavirus disease (SECD) Situation Report

12. Wang L, Byrum B, Zhang Y (2014) Detection and genetic characterization of deltacoronavirus in pigs, Ohio, USA, 2014. Emerg Infect Dis 20:1227-1230

13. Wang L, Byrum B, Zhang Y (2014) Porcine coronavirus HKU15 detected in 9 US states, 2014. Emerg Infect Dis 20:1594-1595

14. Wang L, Zhang Y, Byrum B (2014) Complete genome sequence of porcine coronavirus HKU15 strain IN2847 from the United States. Genome Announc 2. doi:10.1128/genomeA.00291-14

15. Woo PC, Lau SK, Lam CS, Lau CC, Tsang AK, Lau JH, Bai R, Teng JL, Tsang CC, Wang M, Zheng BJ, Chan KH, Yuen KY (2012) Discovery of seven novel Mammalian and avian coronaviruses in the genus deltacoronavirus supports bat coronaviruses as the gene source of alphacoronavirus and betacoronavirus and avian coronaviruses as the gene source of gammacoronavirus and deltacoronavirus. J Virol 86:3995-4008 